\title{
Critical Percolation in High Dimensions
}

\author{
Peter Grassberger \\ John-von-Neumann Institute for Computing, Forschungszentrum Jülich, D-52425 Jülich, Germany
}

(Dated: May 8, 2019)

\begin{abstract}
We present Monte Carlo estimates for site and bond percolation thresholds in simple hypercubic lattices with 4 to 13 dimensions. For $d<6$ they are preliminary, for $d \geq 6$ they are between 20 to $10^{4}$ times more precise than the best previous estimates. This was achieved by three ingredients: (i) simple and fast hashing which allowed us to simulate clusters of millions of sites on computers with less than $500 \mathrm{MB}$ memory; (ii) a histogram method which allowed us to obtain information for several $p$ values from a single simulation; and (iii) a variance reduction technique which is especially efficient at high dimensions where it reduces error bars by a factor up to $\approx 30$ and more. Based on these data we propose a new scaling law for finite cluster size corrections.
\end{abstract}

PACS numbers: 05.10-a, 64.60.A

In spite of decades of intensive studies [1], percolation remains an active subject of research. While there has been enormous progress in understanding percolation in 2 dimensions [2], mainly because of conformal invariance, progress in high dimensions has been much slower. It has been known since long time that $d=6$ is the upper critical dimension [1], and expansions of $p_{c}$ in $1 /(2 d-1)$ have been given already more than 20 years ago. But up to now there exists no detailed numerical study of logarithmic corrections in $d=6$, finite size corrections are not yet understood for $d>6$, and even numerical estimates of $p_{c}$ in $\geq 6$ dimensions are very poor. One reason for this is obviously that straightforward simulations of large lattices in $\geq 6$ dimensions require huge amounts of fast memory. This lack of stimulus by numerical verification certainly was part of the reason for the slow analytical progress.

It is the purpose of the present note to improve on this situation by presenting precise numerical estimates of $p_{c}$ (and of finite cluster size corrections) for site and bond percolation on simple hypercubic lattices with $d=6$ to $d=13$.

Our main results are summarized in Table 1, where we also include preliminary results for $d=4$ and $d=$ 5. We also give the best previous estimates for $p_{c}$ and expansions in $1 /(2 d-1)$. We shall discuss them later in more detail, but here we just point out that our new estimates are vastly better than all previous ones. They were possible, with rather modest effort (we used only fast PCs and Alpha work stations, with altogether ca. $10^{3} \mathrm{CPU}$ hours), due to several important ingredients:

1) We used as basic routine a standard breadth-first version of Leath's algorithm which simulates single clusters. We do not use the popular Hoshen-Kopelman method since that would require prohibitively large memory if we want to simulate large clusters. In Leath's method, one writes the coordinates of each cluster site (which consist of a single integer - see item 2 below) into a first-in-first-out queue $\mathrm{Q}$, where each new entry represents a newly wetted neighbor of the oldest entry in the queue.

2) We used a simple but very efficient form of hashing
[3] for storing the information whether a site has already been wetted or not. On Compaq Alpha work stations with 64 bit long integers, we labelled lattice sites by a single long integer. Using as lattice size $L$ an odd number slightly smaller than $2^{64 / d}$, we label the neighbors of site $i$ as $i \pm 1, i \pm L, \ldots i \pm L^{d-1}$. If we want to simulate bond percolation clusters with roughly $N$ sites, we find first the power of 2 nearest to $N, 2^{k} \approx N$, and use it to obtain for each site $i$ its key $m_{i}=\left(i \bmod 2^{k}\right)($ notice that this is done most efficiently by bitwise AND). Assume now that site $i$ with key $m_{i}$ is the $n$-th site wetted. Then an entry is written into the $m_{i}$-th element of an array of pointers $\mathrm{S}$ of size $2^{k}$. This element points to the $n$-th element of a structure $(\mathrm{L}, \mathrm{Q})$ where $\mathrm{Q}$ is the above queue and $\mathrm{L}$ is a linked list. In $\mathrm{Q}$, the coordinate $i$ is stored. The element of L remains empty, if the key $m_{i}$ had not been encountered before. Otherwise, if some other site $j$ with the same key $m_{j}=m_{i}$ had been wetted in an earlier step $n^{\prime}<n$, the old element of $\mathrm{S}$ (which had pointed to $n^{\prime}$ ) is written in the $n$-th element of L. In this way we can deal with virtual lattices of $2^{64}$ sites, using $2^{k}+2 N_{\max }$ storage places, where $N_{\max }$ is an upper bound on the size of clusters to be simulated. The algorithm is slightly different for site percolation where a tested site has to be excluded from further growth even if it is not wetted, in contrast to bond percolation. It also has to be modified on machines with only 32 bit long integers where a pair of numbers replaces $i$ and a pair of co-prime odd numbers $L_{1}$ and $L_{2}$, both slightly smaller than $2^{32 / d}$, replaces $L$.

This is not as storage efficient as the recent algorithm of [- But it works with usual (pseudo-)random number generators (we used the 4-tap generator with period $2^{9689}-1$ of (5]), while the algorithm of [4 needs a random function generator. The most easily available random function generator today is the Data Encryption Standard [6] which is rather slow when implemented in software and of unproven quality for this application (it was developed for entirely different purposes, and lacks any published theoretical justification).

3) In order to estimate cluster statistics for several values of $p$ from a single run at nominal value $p_{0}$, we use a trick similar to the histogram methods used by 


\begin{tabular}{|c|c|c|c|c|c|c|c|}
\hline & \multicolumn{3}{|c|}{ bond } & \multicolumn{4}{|c|}{ site } \\
\hline & \multirow[b]{2}{*}{ present } & \multicolumn{2}{|c|}{ previous } & \multirow[b]{2}{*}{ present } & \multicolumn{3}{|c|}{ previous } \\
\hline$d$ & & best estimate & \begin{tabular}{|l|} 
Eq.(间) \\
\end{tabular} & & best estimate & Eq.(8) & Eq.(9) \\
\hline 4 & $.1601314(13)$ & $.160130(3)$ 抅 & .15666092 & $.1968861(14)$ & $.196889(3)$ 湖 & .19304456 & .19880605 \\
\hline 5 & $.118172(1)$ & $.118174(4)$ & .11664888 & $.1407966(15)$ & $.14081(1)$ ] & .13793629 & .14004471 \\
\hline 6 & $.0942019(6)$ & $.09420(1)$ & .09365356 & $.109017(2)$ & $.1079(5) \sqrt{9}$ & .10754047 & .10848530 \\
\hline 7 & $.0786752(3)$ & [ & .07847711 & $.0889511(9)$ & $.08893(2) \sqrt{10}$ & .08823220 & .08871655 \\
\hline 8 & $.06770839(7)$ & $.06770(5)$ & .06763062 & $.0752101(5)$ & - & .07485431 & .07512757 \\
\hline 9 & $.05949601(5)$ & $.05950(5)$ & .05946233 & $.0652095(3)$ & - & .06502556 & .06519119 \\
\hline 10 & .05309258(4) & - & .05307663 & .0575930(1) & - & .05749265 & .05759880 \\
\hline 11 & $.04794969(1)$ & - & .04794152 & $.05158971(8)$ & - & .05153203 & .05160316 \\
\hline 12 & $.04372386(1)$ & - & .04371939 & . 046736 & - & .04669616 & .04674559 \\
\hline 13 & $.04018762(1)$ & - & .04018504 & $.04271508(8)$ & - & .04269312 & .04272853 \\
\hline
\end{tabular}

TABLE I: Estimates of $p_{c}$ for bond and site percolation in $d=4$ to $d=13$. Numbers in round brackets are single standard deviations, square brackets refer to the citations at the end of the paper. For $d>9$ the best previous estimates backed by theory were given by the (presumably asymptotic) expansions (7) and (8), while Eq.(9) was a heuristic guess. The estimates for $d=4$ and $d=5$ are preliminary, since we do not yet understand the important corrections to scaling in these cases (all error bars in this paper include plausible worst case estimates of systematic errors).

Dickman [11] for the contact process (see also [12]). If a cluster with $n$ wetted sites and $b$ non-wetted boundary sites was generated with nominal value $p_{0}$, it contributes to the ensemble with $p_{0}$ replaced by $p$ with weight

$$
W=\left(p / p_{0}\right)^{n}\left((1-p) /\left(1-p_{0}\right)\right)^{b} .
$$

Instead of collecting histograms for cluster numbers with fixed $n$ and $b$ (which would have led to excessively large arrays) we calculated on the fly three distributions: One for the nominal $p_{0}$ (which was chosen close to $p_{c}$ as estimated from short test runs and from Eqs.(7) resp. (8)) and two for neighbouring values $p_{ \pm}=p_{0} \pm \delta p$, using Eq.(1) for the latter. Observables at $p$-values in between (including $p_{c}$ ) were obtained by geometric (i.e. linear in logarithm) interpolation. Having three values of $p$ instead of just two allowed us to check that the error due to the interpolation was negligible.

4) Our main observable will be the number $M(t)$ of wetted sites with "chemical distance" $t$ from the seed of the cluster (i.e. the number of sites infected at time $t$, if cluster growth is interpreted as spreading of an epidemic). For $d>6$ we expect its average $\langle M(t)\rangle$ to become a constant at the critical point, since the process is basically a branching process with small corrections. But instead of using $\langle M(t)\rangle$ itself, we obtain a less noisy signal by the following trick which would give the exact ensemble average of $M(t)$ if the cluster growth indeed were a branching process [13].

Let us assume we have a (still growing) cluster $C$ with $M(t)$ sites wetted at step $t$, and denote by $M^{+}(t)$ the number of free neighbors, i.e. the number of sites which can be wetted at step $t+1$. The actual number wetted will fluctuate, but the expected average number, conditioned on $C$ and thus also on $M(t)$, is exactly given by

$$
\mathbf{E}[M(t+1) \mid C]=p M^{+}(t) .
$$

Thus the expected geometric increase of the number of wetted sites, still conditioned on $C$, is

$$
\mathbf{E}[M(t+1) / M(t) \mid C]=p M^{+}(t) / M(t)
$$

and its weighted sample average over all clusters is

$$
r(t) \equiv \frac{\sum_{C} M(t) \mathbf{E}[M(t+1) / M(t) \mid C]}{\sum_{C} M(t)}=\frac{p\left\langle M^{+}(t)\right\rangle}{\langle M(t)\rangle} .
$$

Our estimate for the true ensemble average of $M(t)$ is then finally

$$
\widehat{M(t)}=\prod_{t^{\prime}=0}^{t-1} r\left(t^{\prime}\right) .
$$

Since we measured also the direct estimate $\langle M(t)\rangle$ and the (co-)variances of both estimates, we can also compute the variance of any linear combination of both. For $d=4$ and $d=5$, where both variances are comparable and the covariance is negative, a substantial achievement is obtained by taking as the final estimate the linear combination with the smallest variance.

An expression similar to Eq.(5) can be obtained also for the rms. radius, if we replace the ratios in Eqs.(3 3 by differences and the product in Eq. (5) by a sum. This would also be exact and non-fluctuating if the cluster growth were a branching process with translation invariance.

The variance reduction due to Eq.(5) is largest for small $t$. Yet, for bond percolation in $d=11$, it gave even for the largest $t(=200)$ a factor $\approx 1 / 1000$ over using just $\langle M(t)\rangle$. For $d=6$ and $t=2000$, the reduction was still by a factor $\approx 140$. Indeed, there were substantial improvements even for $d=4$ and 5 , while the improvement in $d=3$ was marginal. For site percolation the improvements were similar but somewhat less dramatic. 


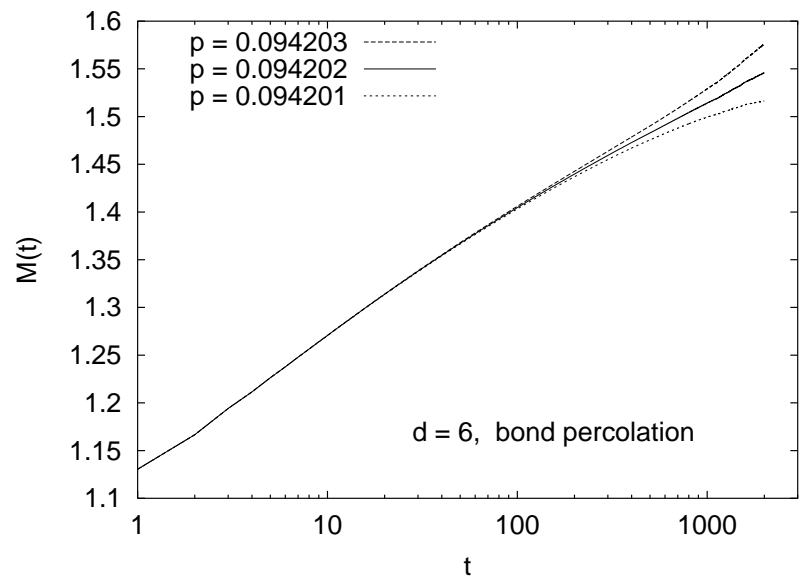

FIG. 1: Plot of $\widehat{M(t)}$ versus $\ln t$ for bond percolation in $d=6$. Statistical errors are smaller than the width of the curves. The main uncertainty in pinning down $p_{c}$ comes from the nonobvious and somewhat subjective extrapolation to $t \rightarrow \infty$.

We should note that we calculated also $P(t)$, the probability that a cluster survives at least $t$ steps (i.e. has "chemical radius" $\geq t$ ), the cluster size distribution $P(n)$, and the spatial extent of clusters with $n$ sites. All of them gave vastly more noisy signals (since we could not use a similar variance reduction trick as for $M(t))$ and were not used in estimating the critical point.

Results for $\widehat{M(t)}$ are shown in Figs. 1 to 3 for $d=6,7$, and 11. In all these figures we show results for bond percolation. Results for site percolation are similar albeit somewhat more noisy. In the first two cases we checked explicitly that no cluster was larger than the virtual lattice size $L$ (which was $>500$ in both cases), so there are strictly no finite lattice size effects. For $d \geq 9$ this was no longer possible for the cluster sizes used here (typically up to $10^{4}-10^{6}$ sites), but we can easily convince ourselves that also there finite size effects are negligible.

In each of the 3 figures the critical point $p_{c}$ is characterized by $d \widehat{M(t)} / d t \rightarrow 0$ for $t \rightarrow \infty$. For $d>6$ we also have $\widehat{M(t)} \rightarrow$ const for $p=p_{c}$, while we see a logarithmic divergence in $d=6$ as predicted by the renormalization group 14 (see Fig.1). Unfortunately, the detailed behaviour of $M(t)$ in $d=6$ has not yet been calculated, though the results of [14] and the fact that $\nu_{t}$ (the exponent controlling the correlation time) is 1 , suggest $M(t) \sim[\log t]^{2 / 7}$ to leading order. Therefore, and since it is notoriously difficult to verify logarithmic terms (see, e.g., 15, 16, 17]), we have not attempted any detailed analysis.

From Figs. 2 and 3 we also see that corrections to scaling decrease strongly with dimension for $d>6$. In Fig.2 we see a straight line for $p=p_{c}$ when plotting $\widehat{M(t)}$ against $1 / \sqrt{t}$, showing that the leading correction term is $\propto t^{-0.5}$ in $d=7$. Similarly, a straight line is obtained for $d=11$ when using $t^{-2.18}$ (Fig.3). All these (and similar results for other values of $d>6$ and for site percolation,

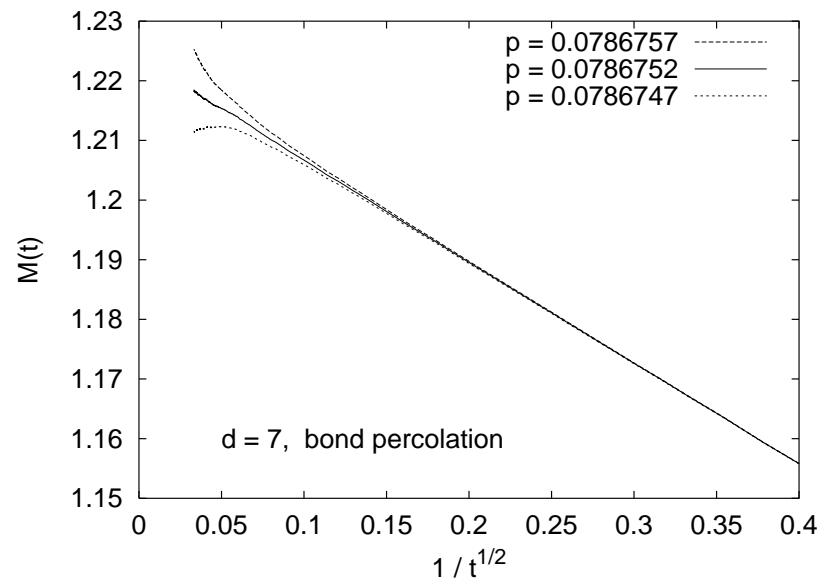

FIG. 2: Plot of $\widehat{M(t)}$ versus $t^{-1 / 2}$ for bond percolation in $d=7$. Statistical errors are always smaller than half the distances between neighboring curves.

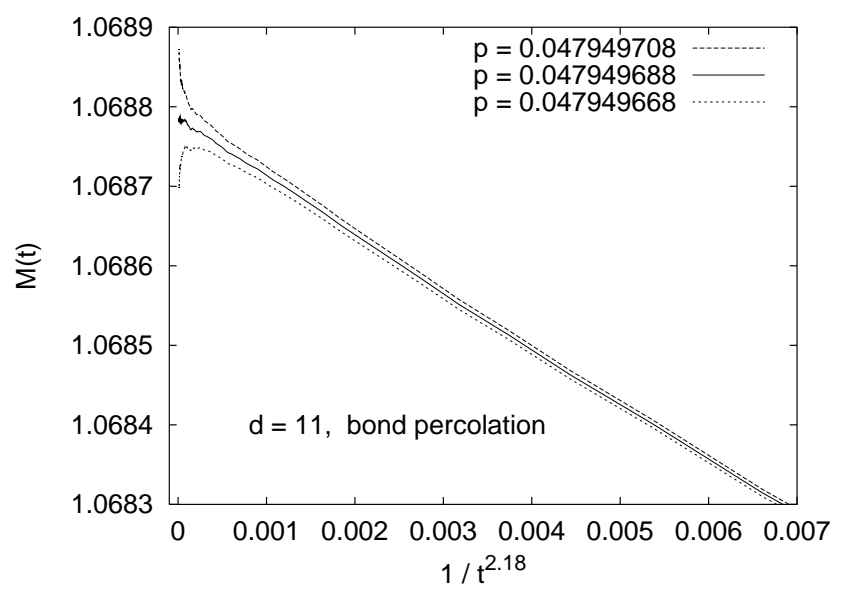

FIG. 3: Plot of $\widehat{M(t)}$ versus $t^{-2.18}$ for bond percolation in $d=11$. Statistical errors are again smaller than half the distances between neighboring curves. The exponent 2.18 is chosen since it gives the straightest line.

not shown here) strongly suggest anomalous scaling

$$
M(t)=M_{\infty}-\text { const } / t^{\omega(d)},
$$

similar to the scaling for self avoiding walks in $d>d_{c}$ found in 177. But while the exponents were simply $(d-$ $\left.d_{c}\right) / 2$ in [17], they seem to depend less trivially on $d$ in the present case - although we cannot exclude the possibility that $\omega(d)=\left(d-d_{c}\right) / 2$ also here, and the observed deviations are due to higher order corrections. The latter is indeed suggested by the results of 18$]$.

The constants $M_{\infty}$ defined in Eq.(6) are plotted in Fig. 4 against $d-6$ on doubly logarithmic scale. They seem to fall on parallel straight lines, suggesting a universal law $M_{\infty}-1 \sim(d-6)^{-a}$ with $a=0.73 \pm 0.03$. But a closer look reveals that deviations from this are significant (although they are small), suggesting that it holds neither for $d \rightarrow \infty$ nor for $d \rightarrow 6$ exactly. 


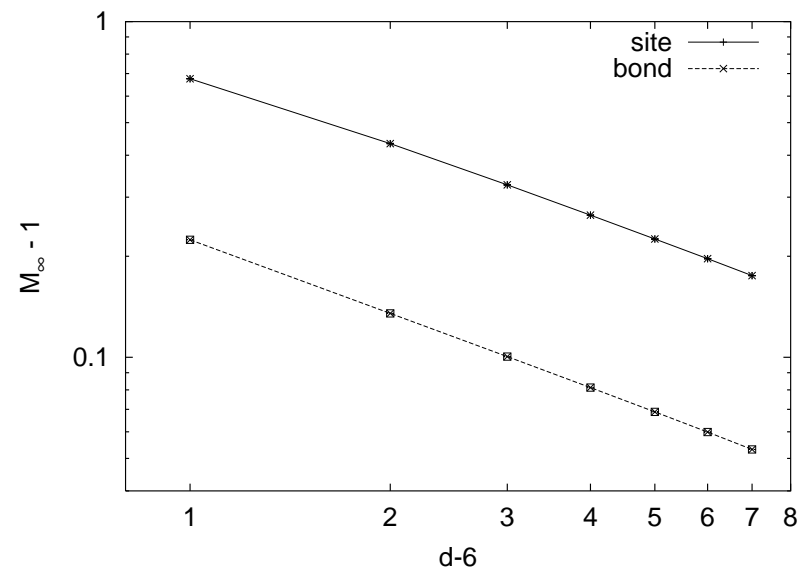

FIG. 4: Log-log plot of $M_{\infty}-1$ against $d-d_{c}$. Statistical errors are smaller than the data symbols.

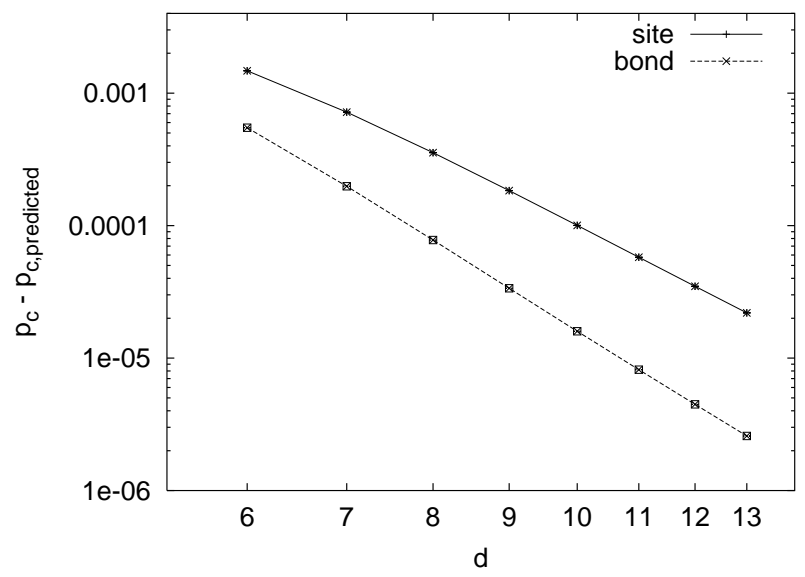

FIG. 5: Log-log plot of the discrepancies between the simulation results and Eqs.(7) and (8). Error bars are smaller than the sizes of the symbols.

Let us finally discuss the $p_{c}$ values given in Table 1 . They should be compared to the predictions [7]

$$
p_{c, \text { bond }}=s+5 s^{3} / 2+15 s^{4} / 2+57 s^{5}+\ldots
$$

and 19]

$$
p_{c, \text { site }}=s+3 s^{2} / 2+15 s^{3} / 4+83 s^{4} / 4+\ldots
$$

with $s=1 /(2 d-1)$. The dots in these equations stand for higher powers of $s$. It was suggested in [19] that they can be approximated, for site percolation at least, by adding $2 / 3$ of the last term,

$$
p_{c, \text { site }} \approx s+3 s^{2} / 2+15 s^{3} / 4+415 s^{4} / 12 .
$$

The full series are presumably only asymptotic. It is thus a priori not clear whether any of these equations should be good approximations to the present data. From Table 1 we see that Eq.(9) is excellent in the range studied here, but it has wrong asymptotic behaviour and should be worse than Eq.(8) for $d \geq 15$. As seen from Fig.5, the agreement with Eqs.(7) and (8) is indeed better than could have been expected: For bond percolation the difference decreases roughly as $s^{7.1}$ (instead of $s^{6}$ ), while for site percolation it decreases as $s^{5.7}$ instead of $s^{5}$. Obviously the next terms in Eqs.(7) and (8) would be needed for a more detailed comparison.

Finally we should remind of several heuristic formulas for $p_{c}$ values on various lattices. All early ansatzes of this type were already refuted in [20] because they contradicted Eqs.(7) or (8). More recently, such heuristics have been discussed again in 21] and in the papers quoted there. We have not attempted any detailed comparison in view of their complete lack of theoretical basis.

In summary, we have presented vastly improved estimates for percolation thresholds on high-dimensional hypercubic lattices. They should be compared to improved series expansions and/or rigorous bounds. At present such results are not available, partly because it had seemed that they could not be compared to any numerical estimates. Apart from this, the methods used in the present paper should also be of use in other similar problems. These include simulations of percolation backbones, conductivity exponents, percolation on more exotic lattices, directed percolation in high dimensions, and self avoiding walks. In all these cases both the hashing and the variance reduction should be of help in simulating larger systems with higher precision.

I thank Walter Nadler and Hsiao-Ping Hsu for discussions and for carefully reading the manuscript.
[1] D. Stauffer and A. Aharony, Introduction to Percolation Theory, (Taylor \& Francis, London 1994).

[2] J. Cardy, preprint math-ph/0103018 (2001).

[3] R. Sedgewick, Algorithms in C (Addison-Wesley, Reading 1990)

[4] G. Paul, R.M. Ziff, and H.E. Stanley, Phys. Rev. E 64, 026115 (2001).

[5] R.M. Ziff, Computers in Physics 12, 385 (1998).

[6] W.H. Press, B.P. Flannery, S.A. Teukolsky, and W.T.
Vetterling, Numerical Recipes (Cambridge Univ. Press, Cambridge 1997).

[7] D.S. Gaunt and H. Ruskin, J. Phys. A 11, 1369 (1978).

[8] J. Adler, Y. Meir, A. Aharony, and A.B. Harris, Phys. Rev. B41, 9183 (1990).

[9] S. van der Marck, J. Phys. A 31, 3449 (1998).

[10] D. Stauffer and R.M. Ziff, Int. J. Modern Phys. C 11, 205 (2000).

[11] R. Dickman, Phys. Rev. E 60, R2441 (1999). 
[12] H.G. Ballesteros et al., Phys. Lett. B 400, 346 (1997).

[13] In a branching process the evolution of each particle and its offspring is independent of the past and of all other particles.

[14] J.W. Essam, D.S. Gaunt, and A.J. Guttmann, J. Phys. A 11, 1983 (1978).

[15] P. Grassberger, Phys. Rev. E56, 3682 (1997).

[16] P. Grassberger, R. Hegger, and L. Schäfer, J. Phys. A 27, 7265 (1994).
[17] A.L. Owczarek and T. Prellberg, J. Phys. A 34, 5773 (2001).

[18] J. Adler, A. Aharony, and A.B. Harris, Phys. Rev. B30, 2832 (1984).

[19] D.S. Gaunt, M.F. Sykes, and H. Ruskin, J. Phys. A 9, 1899 (1976).

[20] D.S. Gaunt and R. Brak, J. Phys. A 17, 1761 (1984).

[21] S. Galam and A. Mauger, Phys. Rev. E 53, 2177 (1996). 